\title{
PERFIL EPIDEMIOLÓGICO DE PESSOAS COM DIABETES MELLITUS ATENDIDAS NAS ESTRATÉGIAS SAÚDE DA FAMÍLIA
}

\section{EPIDEMIOLOGICAL PROFILE OF PEOPLE WITH DIABETES MELLITUS TREATED IN FAMILY HEALTH STRATEGIES}

\section{PERFIL EPIDEMIOLÓGICO DE PERSONAS CON DIABETES MELLITUS ATENDIDAS EN LAS ESTRATEGIAS SALUD DE LA FAMILIA}

\author{
Evandro Dal Soto Busnelo ${ }^{1}$, Claudete Moreschi $^{2}$, Silvana de Oliveira Silva ${ }^{3}$, Sandra Ost \\ Rodrigues $^{4}$, Daina Foggiato de Siqueira ${ }^{5}$, Andiara Luiz Ramos Soares ${ }^{6}$
}

\section{RESUMO}

Objetivo: identificar o perfil epidemiológico das pessoas com diabetes atendidas nas Estratégias Saúde da Família de um município do Rio Grande do Sul. Métodos: estudo quantitativo, transversal. Dados coletados por meio questionários aplicados com 222 pessoas com diabetes mellitus, no período de setembro a outubro de 2018, seguido de análise estatística descritiva. Resultados: perfil predominante do sexo feminino (72,0\%), idade média de 65 anos, ensino fundamental incompleto $(72,5 \%)$, raça branca $(90,1 \%)$, casados $(57,2 \%)$, aposentados/pensionistas $(66,7 \%)$, renda mensal familiar de até um salário. A média de tempo da doença é de 10,1 anos. A maioria é hipertenso (79,7\%), não participa de grupos de educação em diabetes $(87,4 \%)$ e realiza o tratamento com dieta e antidiabéticos orais $(73,9 \%)$. Conclusão: constata-se a importância de promover ações preventivas e de acompanhamento continuo para evitar agravos provenientes do diabetes com vistas à melhorar a qualidade de vida desta população.

Descritores: Diabetes Mellitus; Perfil epidemiológico; Estratégia Saúde da Família.

\footnotetext{
${ }^{1}$ Enfermeiro. Graduado pela Universidade Regional Integrada do Alto Uruguai e das Missões.

${ }^{2}$ Enfermeira. Doutora em Ambiente e Desenvolvimento. Docente do Curso de Enfermagem da URI, Santiago, RS.

${ }^{3}$ Enfermeira. Mestre em Enfermagem. Docente do Curso de Enfermagem da Universidade Regional Integrada do Alto Uruguai e das Missões, Campus Santiago, RS.

${ }^{4}$ Enfermeira. Mestre em Enfermagem. Docente do Curso de Enfermagem da Universidade Regional Integrada do Alto Uruguai e das Missões, Campus Santiago, RS.

${ }^{5}$ Enfermeira. Doutora em Enfermagem. Docente do Curso de Enfermagem da Universidade Regional Integrada do Alto Uruguai e das Missões, Campus Santiago, RS.

${ }^{6}$ Enfermeira. Graduada pela Universidade Regional Integrada do Alto Uruguai e das Missões.
} 


\begin{abstract}
Objective: to identify the epidemiological profile of people with diabetes treated in the Family Health Strategies of a city of Rio Grande do Sul. Methods: a quantitative, cross-sectional study. Data were collected through questionnaires applied with 222 people with diabetes mellitus, from September to October 2018, followed by descriptive statistical analysis. Results: predominantly female $(72.0 \%)$, mean age 65 years, incomplete elementary school $(72.5 \%)$, white race $(90.1 \%)$, married $(57.2 \%)$, retired / pensioners $(66.7 \%)$, monthly family income of up to one salary. The mean duration of the disease was 10.1 years. The majority of them are hypertensive $(79.7 \%)$, do not participate in diabetes education groups $(87.4 \%)$ and carry out treatment with diet and oral antidiabetics (73.9\%). Conclusion: it is important to promote preventive actions and continuous follow-up to avoid diseases from diabetes in order to improve the quality of life of this population.
\end{abstract}

Descriptors: Diabetes Mellitus; Epidemiological profile; Family Health Strategy.

\title{
RESUMEN
}

Objetivo: identificar el perfil epidemiológico de las personas con diabetes atendidas en las Estrategias Salud de la Familia de un municipio de Rio Grande do Sul. Métodos: estudio cuantitativo, transversal. Datos recogidos por medio de cuestionarios aplicados con 222 personas con diabetes mellitus, en el período de septiembre a octubre de 2018, seguido de análisis estadístico descriptivo. Resultados: perfil predominante del sexo femenino $(72,0 \%)$, edad media de 65 años, enseñanza básica incompleta $(72,5 \%)$, raza blanca $(90,1 \%)$, casados $(57,2 \%)$, jubilados / pensionistas $(66,7 \%)$, renta mensual familiar de hasta un salario. El promedio de tiempo de la enfermedad fue de 10,1 años. La mayoría es hipertenso (79,7\%), no participa de grupos de educación en diabetes $(87,4 \%)$ y realiza el tratamiento con dieta y antidiabéticos orales $(73,9 \%)$. Conclusión: se constata la importancia de promover acciones preventivas y de seguimiento continuo para evitar agravios provenientes de la diabetes con miras a mejorar la calidad de vida de esta población.

Descriptores: Diabetes Mellitus; Perfil epidemiológico; Estrategia Salud de la Familia.

\section{INTRODUÇÃO}

O Diabetes Mellitus (DM) é uma doença que aumenta a incidência de acordo com o avanço da idade, com prevalência elevada de morbimortalidade. Esta doença pode apresentar diversas complicações, dentre elas, a insuficiência renal, amputação de membros inferiores, cegueira e doença cardiovascular. O DM é caracterizado por um transtorno metabólico de etiologias heterogêneas, apresentando alteração nos índices da glicose no sangue, decorrente do defeito da secreção e/ou da ação da insulina. ${ }^{1}$

Em 2015, a Federação Internacional de Diabetes estimou que $8,8 \%$ da população mundial com 20 a 79 anos de idade (415 milhões de pessoas) vivia com diabetes. Diante desta tendência, projeta-se que, em 2040, o número de pessoas com diabetes seja superior a 642 milhões. $\mathrm{O}$ aumento da prevalência do diabetes está associado a diversos fatores, dentre eles, transição epidemiológica, transição nutricional, 
rápida urbanização, maior frequência de estilo de vida sedentário, maior frequência de excesso de peso, aumento do envelhecimento populacional e, ainda, à maior sobrevida das pessoas com diabetes. ${ }^{2}$ Por se tratar de uma doença crônica que ainda não possui cura conhecida os profissionais de saúde devem auxiliar as pessoas na prevenção da doença e também na prática do autocuidado. ${ }^{3}$

$\mathrm{O}$ enfermeiro, por estar mais próximo da comunidade, tem um papel de educador e pesquisador, tornando possível o desenvolvimento de atividades que possam esclarecer dúvidas, bem como avaliar as limitações da família e colaborar com a prevenção de agravos. ${ }^{4,5}$

Destaca-se que o enfermeiro possui um papel importante no sentido de prestar um atendimento humanizado às pessoas com diabetes. Este profissional pode contribuir na localização das principais dificuldades relacionadas à adesão ao tratamento, além de orientar acerca da realização de práticas de autocuidado inerentes ao tratamento do DM.

A Estratégia Saúde da Família (ESF) tem um valioso papel na melhora da qualidade de vida dos usuários que possuem DM, pois, por meio da promoção de suas ações, estabelece-se boas práticas a serem seguidas, bem como acompanhamento individualizado de acordo com as necessidades que cada indivíduo apresenta.
Dentre as atividades realizadas pelas ESF, tem-se consultas médicas e de enfermagem até integração com a comunidade através de grupos de educação e atividades que promovam o bem-estar. $^{6}$

Nessa conjuntura, é importante que a equipe de saúde conheça o perfil epidemiológico dos usuários sob sua responsabilidade para planejar e executar ações de cunho gerencial, educativas e assistenciais que atendam eficazmente as necessidades biopsicossociais da população adstrita. Portanto, o presente estudo se justifica na medida em que se propôs conhecer o perfil epidemiológico de pessoas acometidas por DM em um município que aderiu a importantes iniciativas governamentais para qualificar a atenção à saúde como a Planificação da Atenção Básica (PAB) e o Programa Nacional de Melhoria do Acesso e da Qualidade da Atenção Básica (PMAQ). Para o alcance dos objetivos de tais iniciativas a primeira condição é conhecer a realidade sociocultural e sanitária da população e, no município em questão, há carência de tais informações. Frente ao exposto, questiona-se: qual o perfil epidemiológico das pessoas com diabetes atendidas nas Estratégias Saúde da Família de um município do interior do Rio Grande do Sul/RS? O presente estudo teve como objetivo identificar o perfil epidemiológico das pessoas com diabetes atendidas nas 
Estratégias Saúde da Família de um município do Rio Grande do Sul/RS.

\section{MÉTODO}

Trata-se de estudo transversal, descritivo, de abordagem quantitativa, desenvolvido em todas ESF de um município do interior do estado do Rio Grande do Sul, denominado São Francisco de Assis. O mesmo conta com quatro ESF que atendem a população do município.

Participaram da presente pesquisa, um amostra de 222 pessoas com DM cadastradas na área de abrangência das ESF. Esta amostra possui $95 \%$ de confiança e $5 \%$ de erro. Os critérios de inclusão foram pessoas com DM, a partir de 18 anos de idade e residentes na área de abrangência das ESF. Foram excluídas as pessoas não encontradas em seus domicílios após três tentativas de visitas.

A coleta de dados foi realizada no segundo semestre de 2018, entre os meses de setembro a outubro, por meio de um questionário contemplando o perfil sociodemográfico e avaliação clínica, com as seguintes variáveis: idade, sexo/gênero, cor, situação ocupacional/ trabalho, nível de escolaridade, estado civil, renda mensal familiar, número de membros da família que autorreferiram possuir diabetes, tempo da doença, comorbidades, fatores de risco, doenças concomitantes, complicações devido ao diabetes (agudas e crônicas), tipo de tratamento, medicamentos, participação de grupo em educação em diabetes, consultas na ESF devido ao diabetes.

O questionário foi aplicado no âmbito domiciliar, levando em torno de 30 minutos para cada entrevista. Inicialmente, antes da aplicação do questionário, o pesquisador realizou uma aproximação com o serviço de atenção básica do município apresentando o projeto de pesquisa e colheu informações importantes para o desenvolvimento da pesquisa, tais como, os nomes e endereços das pessoas com diabetes cadastradas nas ESF.

Os dados foram organizados em uma planilha do Excel e após foi realizada a análise estatística descritiva e inferencial. As variáveis quantitativas foram descritas através de média e desvio padrão e as variáveis qualitativas através de frequência absoluta e relativa. Os resultados foram apresentados em forma de tabelas.

Para o desenvolvimento da pesquisa, foram observados os aspectos éticos, previstos na Resolução $\mathrm{N}^{\circ} 510$ de 2016 que traz termos e condições a serem seguidos e trata das pesquisas e testes em seres humanos. As diretrizes e normas regulamentadoras estabelecidas na resolução devem ser cumpridas nos projetos de pesquisa envolvendo seres humanos, devendo ainda atender aos fundamentos éticos e científicos, visando à proteção das 
participantes da pesquisa. $^{7}$ Para sua execução, a pesquisa recebeu autorização da Secretaria Municipal de Saúde (SMS) do município. Também recebeu autorização do Comitê de Ética em Pesquisa (COEP) sob número: 2.844.362, e CAAE: 94226818.7.0000.5353.

\section{RESULTADOS}

Ao analisar as variáveis sociodemográficas, foi verificado que a maioria das pessoas com DM são do sexo feminino $(72,0 \%)$, a faixa etária mais prevalente é de 60 a 69 anos $(35,6 \%)$ com média de idade de 65 anos ( $\mathrm{DP}=12,05)$, casados $(57,2 \%)$, com ensino fundamental incompleto (72,5\%), aposentados ou pensionistas $(66,7 \%)$, cor branca $(90,1 \%)$ e renda mensal familiar de até um salário mínimo $(68,0 \%)$ (TABELA 1).

Quanto à ocupação, a maioria $(66,7 \%)$ é aposentado ou pensionista. A cor branca dentre as pessoas com DM prevaleceu em 90,1\%. Quanto à renda mensal familiar, a maioria $(68 \%)$ recebe até um salário (TABELA 1).

Tabela 1: Variáveis sociodemográficas das pessoas com DM atendidas nas ESF de São Francisco de Assis, RS, 2018.

\begin{tabular}{|c|c|c|c|}
\hline Variáveis & Categorias & $\mathbf{n}$ & $\%$ \\
\hline \multirow[t]{2}{*}{ Sexo } & Masculino & 63 & 28,0 \\
\hline & Feminino & 159 & 72,0 \\
\hline \multirow[t]{8}{*}{ Idade } & 0 a 39 & 6 & 2,7 \\
\hline & 40 a 49 & 13 & 5,8 \\
\hline & 50 a 59 & 50 & 22,5 \\
\hline & 60 a 69 & 79 & 35,6 \\
\hline & 70 a 79 & 45 & 20,3 \\
\hline & 80 a 89 & 25 & 11,3 \\
\hline & 90 ou mais & 4 & 1,8 \\
\hline & Total & & \\
\hline \multirow[t]{4}{*}{ Estado Civil } & Solteiro & 33 & 14,9 \\
\hline & Casado & 127 & 57,2 \\
\hline & Viúvo & 48 & 21,6 \\
\hline & Divorciado & 14 & 6,3 \\
\hline \multirow[t]{8}{*}{ Escolaridade } & Não sabe ler nem escrever & 17 & 7,7 \\
\hline & $\begin{array}{l}\text { Ensino fundamental } \\
\text { incompleto }\end{array}$ & 161 & 72,5 \\
\hline & $\begin{array}{l}\text { Ensino fundamental } \\
\text { completo }\end{array}$ & 13 & 5,9 \\
\hline & Ensino médio incompleto & 7 & 3,2 \\
\hline & Ensino médio completo & 17 & 7,7 \\
\hline & Ensino superior incompleto & 3 & 1,3 \\
\hline & Ensino superior completo & 3 & 1,3 \\
\hline & Pós-graduação & 1 & 0,4 \\
\hline Ocupação & Aposentado ou Pensionista & 148 & 66,7 \\
\hline
\end{tabular}




\begin{tabular}{llcc}
\hline Variáveis & Categorias & $\mathbf{n}$ & \% \\
\hline \multirow{4}{*}{ Do lar } & 37 & 16,7 \\
& Agricultor & 6 & 2,7 \\
& Outras & 31 & 13,9 \\
& & & \\
& Branca & 200 & 90,1 \\
& Preta & 5 & 2,3 \\
Renda familiar & Parda & 17 & 7,6 \\
& & & 68,0 \\
& Até 1 salário & 151 & 26,6 \\
& Entre 1 a 2 salários & 59 & 4,1 \\
& Entre 2 a 4 salários & 9 & 1,3 \\
\hline
\end{tabular}

Fonte: Dados da pesquisa (2018)

No estudo das variáveis de avaliação clínica dos participantes, em relação ao número de membros da família que possuem DM, as que não possuem nenhum familiar com DM foram 49,1\%, 30,6\% possuem uma pessoa na família, 9\% duas pessoas e $11,3 \%$ três ou mais pessoas. A média de tempo que os participantes possuem a doença foi de 10,1 anos $(\mathrm{DP}=9,16)$, sendo que a maioria $(60 \%)$ possui DM há mais de cinco anos. Quanto às comorbidades, verificou-se que $79,7 \%$ das pessoas com DM possuem também hipertensão arterial, $39,6 \%$ possuem antecedentes familiares cardiovasculares, $15,3 \%$ são tabagistas, 28,8 consideram-se sedentários e $23,9 \%$ são obesos ou tem sobrepeso. (TABELA 2).

Em relação às complicações decorrentes do DM, 61,3\% responderam que não tiveram e 39,7\% que já. Dentre as complicações agudas, a hiperglicemia apresentou maior percentual com $25,2 \%$ da população. A retinopatia foi à complicação crônica que teve maior prevalência com $33,3 \%$ seguida de cardiopatia com $15,3 \%$. Em relação ao tipo de tratamento, verificouse que a maioria $(73,9 \%)$ realiza com dieta e antidiabéticos orais, $8,1 \%$ fazem uso de dieta e insulina e 16,2\% dieta, antidiabético oral e insulina. $\mathrm{O}$ medicamento mais utilizado no tratamento foi metformina com $83,8 \%$ seguido de insulina com $23 \%$ e glibenclamida com 20,7\%. A maioria $(87,4 \%)$ nunca participou de grupos de educação em diabetes, 36,9\% não buscou atendimento relativo ao diabetes nos últimos doze meses. Dentre os que procuraram atendimento, a maioria $(32,4 \%)$ procurou consulta exclusivamente com o médico, $25,7 \%$ consulta medica e de enfermagem e apenas $4,1 \%$ buscou consulta exclusivamente de enfermagem. (TABELA 2). 
Tabela 2:Variáveis de avaliação clínica das pessoas com DM atendidas nas ESF de São Francisco de Assis, RS, 2018.

\begin{tabular}{|c|c|c|c|}
\hline Variáveis & Categorias & $\mathbf{n}$ & $\%$ \\
\hline \multirow[t]{4}{*}{ Pessoas da família com DM } & Não tem & 109 & 49,1 \\
\hline & 1 & 68 & 30,6 \\
\hline & 2 & 20 & 9,0 \\
\hline & 3 ou mais & 25 & 11,3 \\
\hline \multirow[t]{5}{*}{ Tempo da doença } & $0-5$ & 89 & 40,1 \\
\hline & $6-10$ & 59 & 26,6 \\
\hline & $11-15$ & 36 & 16,2 \\
\hline & 16 anos $>$ & 38 & 17,1 \\
\hline & Total & & \\
\hline \multirow[t]{5}{*}{$\begin{array}{l}\text { Fatores de riscos e doenças } \\
\text { concomitantes }\end{array}$} & $\begin{array}{l}\text { Antecedentes familiares } \\
\text { cardiovasculares }\end{array}$ & 88 & 39,6 \\
\hline & Tabagismo & 34 & 15,3 \\
\hline & Sedentarismo & 64 & 28,8 \\
\hline & Sobrepeso/obesidade & 53 & 23,9 \\
\hline & Hipertensão arterial & 177 & 79,7 \\
\hline \multirow[t]{2}{*}{ Complicações } & Sim & 86 & 38,7 \\
\hline & Não & 136 & 61,3 \\
\hline \multirow[t]{4}{*}{ Complicações agudas } & Hipoglicemia & 27 & 12,2 \\
\hline & Coma hiperosmolar & 3 & 1,4 \\
\hline & Cetoacidose & 0 & 0 \\
\hline & Hiperglicemia & 56 & 25,2 \\
\hline \multirow[t]{5}{*}{ Complicações crônicas } & Retinopatia & 74 & 33,3 \\
\hline & Neuropatia & 5 & 2,3 \\
\hline & Pé diabético & 18 & 8.1 \\
\hline & Nefropatia & 20 & 9,0 \\
\hline & Cardiopatia & 34 & 15,3 \\
\hline \multirow[t]{4}{*}{ Tipo de tratamento } & Dieta & 4 & 1,8 \\
\hline & Dieta e antidiabéticos orais & 164 & 73,9 \\
\hline & Dieta e insulina & 18 & 8,1 \\
\hline & $\begin{array}{l}\text { Dieta, antidiabético oral e } \\
\text { insulina }\end{array}$ & 36 & 16,2 \\
\hline \multirow[t]{4}{*}{ Medicamentos } & Metformina & 186 & 83,8 \\
\hline & Glibenclamida & 46 & 20,7 \\
\hline & Insulina & 51 & 23,0 \\
\hline & Glimepirida & 9 & 4,1 \\
\hline \multirow{2}{*}{$\begin{array}{l}\text { Participação em grupo de } \\
\text { educação em diabetes }\end{array}$} & Sim & 28 & 12,6 \\
\hline & Não & 194 & 87,4 \\
\hline \multirow{4}{*}{$\begin{array}{l}\text { Consulta de } \\
\text { acompanhamento do DM } \\
\text { nos últimos } 12 \text { meses }\end{array}$} & Não consultaram & 82 & 36,9 \\
\hline & $1 \mathrm{vez}$ & 39 & 17,6 \\
\hline & 2 vezes & 51 & 23,0 \\
\hline & 3 ou mais vezes & 50 & 22,5 \\
\hline
\end{tabular}




\begin{tabular}{llcc}
\hline Variáveis & Categorias & $\mathbf{n}$ & \% \\
\hline Profissionais que fizeram o & Enfermeiro & 9 & 4,1 \\
acompanhamento & & 72 & 32,4 \\
& Médico & 57 & 25,7 \\
\hline
\end{tabular}

Fonte: Dados da pesquisa (2018)

\section{DISCUSSÃO}

O presente estudo permitiu constar que, entre as pessoas que possuem DM, há predominância do sexo feminino. Estudos em outras regiões do país evidenciaram resultados semelhantes onde também foi observada a prevalência do sexo feminino. Estudo transversal, desenvolvido no Estado de Piauí com pessoas com DM cadastradas em Unidades Básicas de Saúde também constatou prevalência de mulheres $(72,6 \%) .{ }^{8}$ Estudo realizado em duas farmácias particulares no município de Itabaiana/Sergipe com pessoas com DM que procuram drogarias particulares para obtenção de medicamentos e acompanhamento do agravo também evidenciou predominância do sexo feminino $(66,6 \%) .{ }^{9} \quad$ Esta constatação também foi encontrada em pesquisa que analisou o perfil epidemiológico de pessoas com diabetes atendidas nas ESF de um município do estado do Rio Grande do Sul, a qual confirmou que esta população em sua maioria é de mulheres $(68,0 \%) .{ }^{10}$ Esses dados reforçam a tendência da população mundial ter por sua maioria pessoas do sexo feminino. ${ }^{11}$ Reflete-se que o fato das mulheres procurarem mais os serviços de saúde pode contribuir com a predominância feminina de pessoas com DM, de acordo com os achados deste estudo.

A média de idade da população deste estudo foi de 65 anos de idade e a maior prevalência foi entre a idade de 50 a 69 anos. A média é compatível com dados de outros estudos que mostram a predominância da doença em pessoas com mais de 55 anos. A predominância desta faixa etária pode ser explicada pelo fato do DM ser uma doença crônica que possui maior prevalência em idosos..$^{9}$ Ao avaliar a prevalência e o perfil das pessoas com DM, autorreferidas no Sistema de Informação da Atenção Básica (SIAB), do município de Lajeado/RS, no período de 2011 a 2013, constatou-se que, na sua maioria, esta população estava acima de 60 anos $(60 \%$ em 2011, 58\% em 2012 e $60 \%$ em 2013). ${ }^{10}$

Quanto ao estado civil, percebeu-se que a maioria é casado, indo ao encontro de dados observados em outros trabalhos que confirmam que a maioria das pessoas que possuem DM é casada..$^{8,11,12} \mathrm{O}$ estado civil pode influenciar na adesão ao autocuidado, visto que nas interações familiares as 
pessoas encontram apoio para 0 enfrentamento de suas condições.

O presente estudo encontrou que a escolaridade predominante é ensino médio incompleto. Isto também foi visto em outros estudos os quais evidenciam baixa escolaridade na população diabética. ${ }^{8-10,12} \mathrm{~A}$ baixa escolaridade tende a influenciar na adesão ao tratamento, o que eleva a reponsabilidade da ESF em relação ao controle da doença. ${ }^{11}$

Em relação à ocupação 66,75\% declararam-se aposentados/ pensionistas, de acordo com outra pesquisa que indica uma maioria de pessoas aposentadas entre os diabéticos. ${ }^{9}$ Em uma pesquisa no Piauí foi encontrado um total de $44,5 \%$ de aposentados, sendo essa a principal fonte de renda dos entrevistados. ${ }^{8}$

A respeito do tempo de convívio com a doença, a maioria possui há mais de cinco anos, resultado esse que se assemelha a um estudo realizado com idosos em Pernambuco que também encontrou-se uma maioria de pessoas com essa faixa de tempo. ${ }^{13}$ Entretanto, foi divergente de um estudo realizado em Minas Gerais onde se observou que $35,2 \%$ tinham DM de 5 a 10 anos. ${ }^{12}$ Frente a estes achados, acredita-se que se as pessoas estão convivendo há mais tempo com o DM é porque estão se cuidando e sabendo conviver com as limitações e cuidados necessários inerentes ao DM. Tal situação pode ocorrer em decorrência da efetividade de um trabalho eficaz da atenção básica.

Sobre o tabagismo foi possível verificar um baixo índice de tabagistas (15,3\%), o que está em concordância com um estudo feito com diabéticos do tipo I e II no nordeste brasileiro o qual evidenciou porcentagem nos dois grupos 17,2\% e $17,7 \%$, respectivamente. ${ }^{14}$ Ressalta-se que este estudo não analisou grupos separados.

A porcentagem de $28,8 \%$ encontrada na pesquisa em relação ao sedentarismo, quando comparado a outros estudos obteve semelhança em um e divergência em outro. Em uma pesquisa realizada com diabéticos hipertensos encontrou-se $27,8 \%$ de pessoas sedentárias. ${ }^{11}$ Já em outro estudo realizado no nordeste brasileiro, onde havia distinção entre grupos de diabetes do tipo I e II obteve-se $30,8 \%$ no grupo de diabetes tipo I e $42,9 \%$ no grupo de pessoas de diabetes tipo II. $^{14}$ Infere-se que o sedentarismo é prejudicial ao controle glicêmico, no entanto, destaca-se que a equipe de saúde da ESF pode promover ações de cuidado visando a adesão de hábitos e estilos de vida que possam diminuir o percentual de pessoas sedentárias e consequentemente diminuir as taxas glicêmicas e melhorar a qualidade de vida da população que possui DM.

Quanto ao tratamento seguido, observou-se um maior uso de antidiabéticos 
orais, seguido do uso de insulina. Uma pesquisa realizada na região nordeste do país obteve resultados semelhantes, identificando que $86,0 \%$ da população faz uso de antidiabéticos orais e $15,9 \%$ uso de insulina. ${ }^{12}$

De acordo com o Ministério da Saúde, houve a adoção de um padrão no tratamento do DM, utilizando medicamentos preconizados pela organização mundial da saúde. Os hipoglicemiantes glibenclamida e metformina juntamente com insulina NPH 100UI passaram a ser disponibilizados na rede de saúde pública. ${ }^{15}$ Os antidiabéticos orais costumam ser a primeira escolha no tratamento do DM, os quais possuem boa adaptação em seu uso além da possibilidade da utilização em associação com outros medicamentos no auxílio da queda da glicemia. ${ }^{1}$

Quando há a necessidade do uso de medicamentos orais para o tratamento da DM, o tratamento preferencial é por meio do uso de metformina. A ação deste medicamento aumenta a captação de glicose no sistema musculoesquelético, diminui a resistência à insulina e reduz a produção de glicose pelo fígado. Quando o uso de apenas metformina não está sendo eficaz usa-se a associação com outro fármaco que normalmente são as sulfonilureias, que agem sobre as células beta na secreção de insulina e na redução dos níveis de glicose no plasma. ${ }^{1}$

Verificou-se uma baixa adesão dos usuários diabéticos em grupos de educação, com 87,4\%, afirmando nunca ter participado desees grupos de educação. Em estudo realizado em Pernambuco, observou-se baixa adesão de participação em grupos, indicando que $79,3 \%$ não participavam. ${ }^{16}$ Os grupos de educação mostram-se como estratégias influenciadoras na melhora da qualidade de vida. Através da troca de saberes há a possibilidade de reflexão sobre assuntos pertinentes, empoderamento e incentivo na autonomia dos participantes. ${ }^{17}$

Sobre a baixa adesão aos grupos de educação em diabetes torna-se possível refletir que apesar dos participantes terem acesso aos grupos, observou-se que eles não comparecem. Essa pouca adesão aos grupos pode ocorrer pelo fato deles não se sentirem motivados a participarem. Frente a isso, entende-se que os profissionais de saúde das ESF precisam repensar sobre as metodologias de condução das atividades educativas ofertadas nos grupos, de forma que estes sintam-se instigados a participarem dos grupos e aderirem as práticas de autocuidado em diabetes visando a efetividade do tratamento, prevenção de complicações decorrentes do DM e melhoria da qualidade de vida. Acredita-se que os grupos de educação 
mostram-se como estratégia influenciadora na melhora da qualidade de vida das pessoas com DM. Estes momentos representam espaços de troca de saberes e possibilidade de reflexão sobre assuntos pertinentes ao tratamento e prevenção do DM, bem como como ao empoderamento e incentivo na autonomia dos indivíduos.

O dado referente à consulta de acompanhamento da DM nos últimos 12 meses indica que das pessoas cadastradas, $36,9 \%$ não realizaram a consulta. Reportando-se à Planificação da Atenção Básica e ao PMAQ, esse dado indica a necessidade das equipes de saúde organizarem a atenção à saúde das pessoas com condições crônicas por meio de ações proativas, contínuas, programadas e integrais. Essa organização perpassa ainda pela necessidade de estratificar o risco à que a pessoa está exposta, o que significa considerar além dos aspectos biológicos também os determinantes sociais da saúde. $^{18}$

Vale destacar ainda que os dados deste estudo revelaram a incipiente atuação do enfermeiro no acompanhamento das pessoas com DM. No entanto, acredita-se no potencial da consulta de enfermagem como um recurso para produzir efeitos positivos no controle da DM e na melhoria da qualidade de vida dos usuários. Cabe ao enfermeiro despertar a autonomia da pessoa com DM, por meio da educação em saúde e cuidado compartilhado, como tecnologias para a promoção do cuidado e prevenção de agravos, com vistas ao protagonismo da pessoa que convive com a condição crônica. 19

\section{CONCLUSÃO}

Ao analisar as variáveis sociodemográficas e das pessoas com DM atendidas nas ESF de um município do Rio Grande do Sul, constatou-se que a maioria desta população é do sexo feminino, idosa, casada, branca, na faixa etária de 59 a 69 anos de idade, não concluíram o ensino médio, aposentados ou pensionistas.

Em relação às variáveis clínicas, observou-se que a maioria possui outro familiar com a mesma doença $(51,9 \%)$. A maioria convive com o DM por mais de 6 anos. Os fatores de risco e doenças concomitantes que mais evidenciaram-se foram hipertensão arterial e antecedentes familiares cardiovasculares, respectivamente. As complicações crônicas mais citadas foram hiperglicemia e hipoglicemia. As complicações agudas predominantes foram retinopatia e cardiopatia respectivamente. O tipo de tratamento predominante foi dieta e uso de antidiabéticos orais. Os medicamentos citados com maior frequência foram metiformina, insulina e glibenclamida, respectivamente. A maior parte não 
participou em grupos de educação em diabetes. Entre os que buscaram atendimento, a maioria consultou com o médico, seguido do enfermeiro.

A partir dos resultados encontrados neste estudo foi possível observar a importância das ações preventivas e do acompanhamento continuo da evolução da doença, para evitar agravos da DM, bem como buscar uma melhora na qualidade de vida. É necessário levar em consideração o meio de onde o indivíduo está inserido para que essas mudanças possam ser efetivas e de acordo com a sua realidade. Considerase como limitação do estudo o fato deste ter sido desenvolvido em apenas um município do Estado do Rio Grande do Sul Sugere-se, portanto, que sejam realizados estudos multicêntricos contemplando uma população como maior abrangência.

\section{REFERÊNCIAS}

1. Brasil. Ministério da Saúde. Conselho Nacional de Saúde. Ética na Pesquisa na área de Ciências Humanas e Sociais.

Resolução N 510, de 07 de abril de 2016. Brasília: CNS, 2013.

2. Brasil. Sociedade Brasileira de Diabetes. Diretrizes da Sociedade Brasileira de Diabetes 2017-2018. Organização José Egídio Paulo de Oliveira, Renan Magalhães Montenegro Junior, Sérgio Vencio. São Paulo: Editora Clannad, 2017. 3. Oliveira DM, Scholeller SD, Hammerschmidt KA, Silva DMGV, Arruda C. Desafios no cuidado às complicações agudas do diabetes mellitus em serviço de emergência adulto. Rev.
Eletr. Enf. [Internet]. 2016 [citado em: 10/06/2018];18:e1163. Doi: http://dx.doi.org/10.5216/ree.v18.35523 4. Barbiani R, Dalla Nora CR, Schaefer R. Nursing practices in the primary health care context: a scoping review. Rev. Latino-Am. Enfermagem. [Internet]. 2016 [citado em: 10/06/2018];24:e2721. Doi: http://dx.doi.org/10.1590/1518-8345.0880.2721 5. Jéssica Mazutti Penso JM, Périco E. Mortalidade por diabetes mellitus em uma macrorregião de saúde de Minas Gerais. Rev Enferm Atenção Saúde [Internet]. 2016 [citado em: 10/12/2018];5(2):34-44. Disponível em: http://seer.uftm.edu.br/revistaeletronica/ind ex.php/enfer/article/view/1536/pdf 6. Moreschi C, Rempel C, Backes DS, Pombo CNF, Siqueira DF, Pissaia LF. Ações das equipes da esf para a qualidade de vida das pessoas com diabetes. Cienc Cuid Saude. [Internet]. 2018 [citado em: 15/12/2018];17(2):1-8. Disponível em: http://periodicos.uem.br/ojs/index.php/Cie ncCuidSaude/article/view/41000/75137513 8151

7. Brasil. Ministério da Saúde. Conselho Nacional de Saúde. Ética na Pesquisa na área de Ciências Humanas e Sociais.

Resolução N 510, de 07 de abril de 2016. Brasília: CNS, 2016.

8. Lima Neto JCG, Oliveira JFSF, Souza MA, Araújo MFM, Damasceno MMC, Freitas RWJF. Prevalencia del síndrome metabólico y sus componentes en personas con diabetes mellitus tipo 2. Texto Contexto Enferm. [Internet]. 2018 [citado em: 15/12/2018]; 27(3):e3900016. Doi: http://dx.doi.org/10.1590/0104$\underline{070720180003900016}$

9. Gois CO, Lima SVMA; Santos ACFS, Melo HN. Perfil dos portadores de diabetes mellitus atendidos em farmácias particulares de Sergipe, Brasil. Scientia Plena [Internet] 2017 [citado em: 15/12/2018]; 13 (1):1-7. Disponível em: https://www.scientiaplena.org.br/sp/article/ viewFile/3764/1869

10. Moreschi, C; Rempel C; Carreno I; Silva DS; Pombo C.NF. Cano, M.R.L. 
Prevalência e perfil das pessoas com diabetes cadastradas no sistema de informação da atenção básica (SIAB). Rev Bras Promoç Saúde [Internet] 2015 [citado em: 16/12/2018]; 28(2): 184-90.Disponível em:

https://periodicos.unifor.br/RBPS/article/vi ew/3598

11. Miranzi SSC, Ferreira FS, Iwamoto HH, Pereira GA, Miranzi MAS. Qualidade de vida de indivíduos com diabetes mellitus e hipertensão acompanhados por uma equipe de saúde da família. Texto Contexto Enferm, [Internet] 2008 [citado em: 11/12/2018]; 17(4): 672-9. Disponível em: http://www.scielo.br/pdf/tce/v17n4/07 12. Santos I, Guerra RG, Silva LA. Características individuais e clínicas de pessoas idosas com diabetes: investigação temáticaemoficina sociopoética. Rev. enferm. UERJ [ Internet] 2013 [citado em: 10/12/2018];21(1):34-40. Disponível em: http://www.facenf.uerj.br/v21n1/v21n1a06 .pdf

13. Amorim TC, Burgos MGPA, Cabral PC. Perfil clínico e antropométrico de pacientes idosos com diabetes mellitus tipo 2. Sci Med. [Internet] 2017 [citado em: 10/12/2018]; 27(3):1-15. Disponível em: https://www.researchgate.net/publication/3 18669348_Perfil_clinico_e_antropometric o_de_pacientes_idosos_com_diabetes_mel litus_tipo_2_atendidos_em_ambulatorio 14. Filho ACAA; Almeida PD; Araújo AKL; et al. Sales IMM; Araújo TME, Rocha SS. Perfil epidemiológico do Diabetes Mellitus em um estado do nordeste brasileiro. J. res.: fundam. care. Online [Internet] 2017 [citado em: 10/10/2018]; 9(3):1-15. Disponível em: https://www.redalyc.org/pdf/5057/5057541 16006.pdf

15. Brasil. Ministério da Saúde. VILA-

NOVA, R.S. Coordenação Nacional de HA e DM. Departamento de Atenção Básica. Secretaria de Atenção à Saúde. 2011.

16. Santos EMS, Souza VP, Correio IAG, Correio EBS. Autocuidado de Usuários Com Diabetes Mellitus: Perfil

Sociodemográfico, Clínico e Terapêutico.
Rev Fund Care Online. [Internet] 2018 [citado em: 10/10/2018];10(3):720-728. Disponível em:

http://www.seer.unirio.br/index.php/cuidad ofundamental/article/viewFile/6180/pdf 1 17. Ritter CB, Aires M, Rotolli A, Santos JLG. Grupo como tecnologia assistencial para o trabalho em enfermagem na saúde coletiva. Sau. \& Transf. Soc. 2014;5(3):8390.

18. Mendes EV. Entrevista: A abordagem das condições crônicas pelo Sistema Único de Saúde. Ciência \& Saúde Coletiva [Internet].2018 [citado em: 10/01/2019]; 23(2):431-435. Disponível em: http://www.scielo.br/pdf/csc/v23n2/14138123-csc-23-02-0431.pdf 19. Silva SO, Machado LM, Schimith MD, Silva LMC, Silveira VN, Bastos AC. Nursing consultation for people with diabetes mellitus: experience with an active methodology. Rev Bras Enferm [Internet]. 2018 [citado em: 10/12/2018];71(6):3103-8. DOI: http://dx.doi.org/10.1590/0034-7167-2017$\underline{0611}$

RECEBIDO: 16/02/2019

APROVADO: 01/10/2019

PUBLICADO: $12 / 2019$ 